\title{
Consensus Folding of Unaligned RNA Sequences Revisited
}

\author{
VINEET BAFNA, ${ }^{1}$ HAIXU TANG, ${ }^{2}$ and SHAOJIE ZHANG ${ }^{1}$
}

\begin{abstract}
As one of the earliest problems in computational biology, RNA secondary structure prediction (sometimes referred to as "RNA folding") problem has attracted attention again, thanks to the recent discoveries of many novel non-coding RNA molecules. The two common approaches to this problem are de novo prediction of RNA secondary structure based on energy minimization and the consensus folding approach (computing the common secondary structure for a set of unaligned RNA sequences). Consensus folding algorithms work well when the correct seed alignment is part of the input to the problem. However, seed alignment itself is a challenging problem for diverged RNA families. In this paper, we propose a novel framework to predict the common secondary structure for unaligned RNA sequences. By matching putative stacks in RNA sequences, we make use of both primary sequence information and thermodynamic stability for prediction at the same time. We show that our method can predict the correct common RNA secondary structures even when we are given only a limited number of unaligned RNA sequences, and it outperforms current algorithms in sensitivity and accuracy.
\end{abstract}

Key words: RNA secondary structure prediction, RNA consensus folding, RNA stack configuration, dynamic programming.

\section{INTRODUCTION}

W ITH THE RECENT DISCOVERY OF NOVEL NONCODING RNA (ncRNA) families (Argaman et al., 2001; Stormo, 2003), RNA is rapidly gaining importance as a molecule of interest (Eddy, 2001; Storz, 2002). A recent article puts the number of human genes down to about 20,000-25,000 (International, 2004). By comparison, even the worm C. elegans has around 19,500 genes. On the other hand, expression activity has been detected on a much larger portion of the human genome (Cawley et al., 2004; Kampa et al., 2004). It is likely that many of these novel transcripts are ncRNA genes, which may carry on many unknown cellular functions. Like proteins, RNA structures are more important for function than are their sequences: RNAs with similar functions often have similar structures but distinct primary sequences. Therefore, understanding the structures of these RNA molecules will help elucidate their functions. Consider the recent

\footnotetext{
${ }^{1}$ Department of Computer Science and Engineering, University of California, San Diego, La Jolla, CA 92093.

${ }^{2}$ School of Informatics and Center for Genomics and Bioinformatics, Indiana University, Bloomington, IN 47408 .
} 
exciting discovery of riboswitches (Nahvi et al., 2003; Vitreschak et al., 2003) as an example. These control elements, with a conserved secondary structure, are located in the untranslated regions of genes coding for proteins that are involved in variant metabolite (nucleic acids, amino acids, etc.) synthesis pathways. The riboswitches can turn off the expression of their downstream genes by binding to certain metabolites and subsequently changing their secondary structures and blocking the translation initiation.

While there is a resurgence in interest in ncRNA, the problem of RNA secondary structure prediction has been extensively studied since the 1970s. The key idea is that to stabilize its structure, distant base pairs in the single-stranded RNA molecule must form hydrogen bonds. There are two distinct approaches to predict RNA secondary structure. The RNA folding approach, initiated by Tinoco et al. (1971), assigns free energies to the components of RNA secondary structure and then computes the RNA secondary structure with the minimum energy. Dynamic programming algorithms have been developed to compute minimum energy secondary structures (Nussinov et al., 1978; Smith and Waterman, 1978; Waterman, 1978; Nussinov and Jacobson, 1980; Zuker and Sankoff, 1984) and implemented in software packages such as MFOLD (Hofacker et al., 1994; Hofacker, 2003) and ViennaRNA (Zuker and Stiegler, 1981; Zuker and Sankoff, 1984; Zuker, 1994, 2003). However, RNA folding via energy minimization has its shortcomings. First, fold prediction depends critically upon correct values of the energy parameters, as shown by Jaeger et al. (1989), which are hard to obtain experimentally. Also, RNA folding in a real cell is mediated by interactions with other molecules, and the absence of knowledge of these interactions may cause misfolding in silico. Pavesi et al. (2004) tried to alleviate this problem by comparing minimum energy structures of a set of RNA sequences from the same family to determine conserved secondary structure. However, it is unclear how the misprediction of secondary structure for a single RNA sequence can affect the accuracy of this approach.

A different approach attempts to resolve these shortcomings by using evolutionary conservation of structure as the basis for structure prediction. It needs as input multiple RNA sequences from an RNA family that have common secondary structures. Since for divergent sequences, the mutations in base pairing regions must be compensated in the complementary base to preserve structure, the presence of multiple covarying mutations is a strong signal for base pairing. In fact, most RNA sequences are selected more for maintenance of the structure than conservation of primary sequence. If the sequence similarity between the given RNA sequences is appropriate, one can first align these sequences using a multiple sequence alignment algorithm and then figure out the potential base pairs in RNA secondary structures by looking at regions with a high number of compensating mutations. Levitt successfully derived the theoretical tRNA secondary structure using this approach, which was largely confirmed by crystallography (Levitt, 1969), and various other structures have been determined through such analysis. Computer programs were implemented later on to achieve this goal automatically (Hofacker et al., 2002).

However, aligning multiple and divergent RNA sequences so as to preserve their conserved structures is not easy, because many compensatory mutations decrease the overall sequence similarity. For unaligned sequences, one must compute the structure and alignment simultaneously. Sankoff proposed an algorithm that can simultaneously align RNA sequences and find the optimal common fold (Sankoff, 1985; Gorodkin et al., 2001; Mathews and Turner, 2002). However, the complexity of this algorithm is $O\left(l^{6}\right)$, where $l$ is the length of RNA sequences, too high to be practical even for two sequences. The complexity can be reduced to $O\left(l^{4}\right)$ (Gorodkin et al., 1997), but only when RNA has no multiloop structure. Eddy and Durbin, and other groups (Eddy and Durbin, 1994; Sakakibara et al., 1994; Knudsen and Hein, 2003) pioneered the approach of modeling RNA sequences using stochastic context free grammars. The rules of the SCFG allow for position dependent scoring of distant base pairs and primary sequence conservation, and also allow automated estimation of model parameters from unaligned sequences using EM. However, in practice, the extensive divergence of RNA sequences makes it hard to reconstruct structure and alignment with perfect accuracy, and the covariance models work best when supplied with good seed alignments. Much recent work has focused on improving fold prediction for aligned sequences (Hofacker et al., 2002; Knight et al., 2004).

In our approach to this well-researched problem, we are motivated by the idea of constraining allowed folds to make it more likely to reach the final correct structure. This idea has been used with good success in aligning divergent genomic sequences. For diverged DNA sequences, there are not enough signals for probabilistic models such as HMMs to be effective without prior information (in the form of seed alignments). The recent methods (Bray and Pachter, 2004; Brudno et al., 2004; Lippert et al., 2004) 
identify anchors corresponding to highly conserved orthologous regions and use these to constrain the multiple alignments. This approach has been used in RNA as well. Waterman (1989) pioneered this with a statistical approach for choosing conserved stem-loops within a pair of fixed-size windows in a set of unaligned RNA sequences. Ji et al. (2004) extended this idea considerably. Starting with putative stemloops, they remove all but the ones conserved in a global sequence alignment of two sequences. These are further culled to retain only those that are present in every sequence in the family. Perriquet et al. (2003) proposed a different anchoring approach to solving the same problem by first determining anchor regions that are highly conserved in given RNA sequences and then seeking a set of conserved stems crossing the same anchor regions that have minimum folding energy. Both methods use primary sequence conservation extensively and limit the variability in the length of loop regions, which may lead to accurate but relatively few predicted anchor stacks for diverged families. On the contrary, Bouthinon and Soldano (1999) and Davydov and Batzoglou (2004) each proposed algorithms to select conserved base pairs among the given RNA sequences based solely on the conservation of the structure that they form, considering neither their sequence similarity nor the thermodynamic stability of this structure. As a result, these methods may risk selecting wrong base pairs when only a limited number of RNA sequences are given.

In this paper, we describe a method, RNAscf (RNA stacks based consensus folding), for predicting the consensus fold of an RNA family, given unaligned sequences. Our method is based on the notion of finding structurally conserved anchors and an iterative extension constrained by the anchors. With relatively few parameters and limited training, the method outperforms other competing methods (see Results), detecting $88 \%$ of true stacks (sensitivity), and overlapping with a correct stack in $93 \%$ of all predictions. The method establishes the validity of this new paradigm for computing consensus structure in RNA. We also mention a possible extension based on an iterative refinement of the predicted structure (see Discussion).

\section{APPROACH}

As shown in Fig. 1a, the secondary structure of an RNA has a tree-like shape. Assume that there is a dummy base pair between 0 and $n+1$. Define a loop as a set of indices $i_{1} \leq i_{2} \ldots \leq i_{k}$ such that for all $j$, either $i_{j+1}=i_{j}+1$, or $\left(i_{j}, i_{j+1}\right)$ form a base pair. Further, if for some $j, j^{\prime},\left(i_{j}, i_{j^{\prime}}\right)$ form a base pair, then $\left|j-j^{\prime}\right|=1$. It can be seen that the structure can be decomposed uniquely into a set of loops, and the loops can be classified as hairpin (containing only one base pair), a stem-loop (two base pairs with no unpaired bases), an interior loop/bulge (two base pairs with unpaired bases), and a multiloop ( $k>2$ base pairs). Figure $1 b$ provides a "stretched" view. of the RNA structure.

The stability of the RNA structure is determined predominantly by stacks of consecutive stem-loops. The stacks are stabilized by hydrogen bonds between the base pairs, and in general, the longer a stack region, the more energetically favorable it is. Each stack corresponds to a pair of substrings. These pairs are typically noninterleaving. While interleaved stacks, or pseudoknots (such as the pair $\left(f, f^{\prime}\right)$ and $\left(h, h^{\prime}\right)$

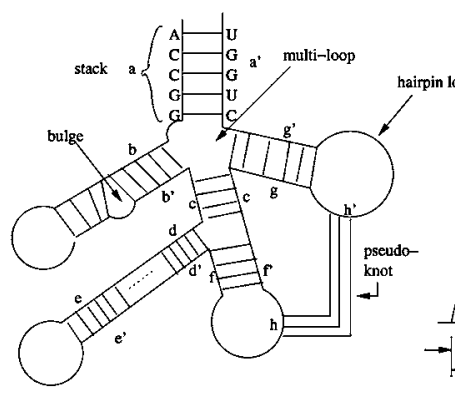

(a)

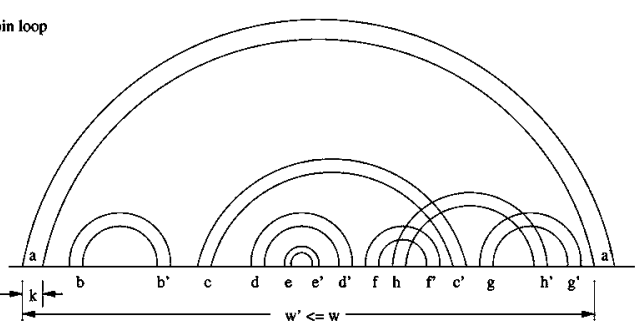

(b)

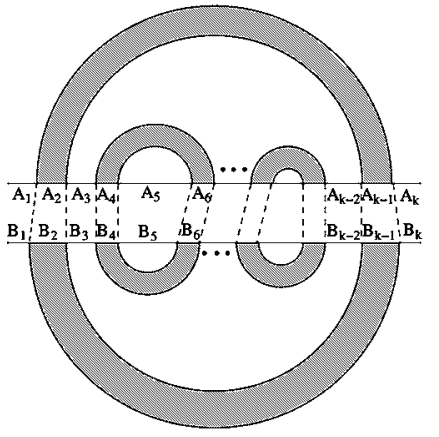

(c)

FIG. 1. (a) An RNA secondary structure with various structural elements including stacked stem-loops, bulges, hairpins, and multiloops. (b) An alternative view of RNA secondary structure in (a). (c) Two stack configurations matching each other for both unpaired regions and paired regions. 
in Fig. 1a) do occur, they are relatively less common and ignored here. In our approach for finding anchors, we ignore individual base pairs and work with a slightly generalized notion of a stack that includes unpaired bases. Configurations of stacks that are conserved in multiple sequences will be the anchors in determining consensus structures.

\subsection{Predicting putative stacks}

The thermodynamic stability of a stack is proportional to the number of hydrogen bonds between the base pairs in the stack. Any pair of strings can be aligned (with gaps), so as to optimize the energy of the paired bases. Therefore, given an RNA string $A$, we construct a local alignment of $A[1, \ldots, n]$ with $A[n, \ldots, 1]$. Let $\delta_{h}(i, j)$ be the score (number of h-bonds) in an $(A[i], A[j])$ base pairing. Thus base pairing of G-C, A-T, G-U is scored 3,2 , and 1 , respectively. Let $S[i, j]$ be optimum score for a stack with left end-point $i$, and right end-point $j$. Then

$$
S[i, j]=\max \left\{\begin{array}{l}
S[i+1, j-1]+\delta_{h}(i, j) \quad\left(\delta_{h}[i, j]>0\right) \\
S[i+1, j]+g \\
S[i, j-1]+g \\
0
\end{array}\right.
$$

where $g$ is a gap penalty. In our implementation, we modify this basic approach to include affine gap costs. We select each $(i, j)$ for which $S[i, j]$ is greater than some threshold. In order to avoid predicting overlapping stacks, we sort the stacks by decreasing score values. Each time a stack is picked, all base pairs in it are excluded. While straightforward, this is an effective procedure. Intuitively, the probability of finding a base pair at random is much higher than the probability of finding a high-scoring stack. Waterman showed that finding a k-long stack within certain window size in many given sequences (even if not all) can be significant (Waterman, 1989). Also, most real base pairs in correct structures should be stabilized by multiple stacked base pairs, implying that limiting consideration to high-scoring stacks does not results in many false negatives.

To test this, we computed statistics on the seed alignments in the RFAM database (Griffiths-Jones et al., 2003). All stacks from seed alignments of 379 families (9,559 sequences) were selected. The seed alignment contains known representative members of the family, which is hand-curated and is annotated with structural information. To correct for annotation errors, the stacks were realigned to each other locally and extended so as to be locally maximal without unpaired bases. Figure 2 describes plots the cumulative

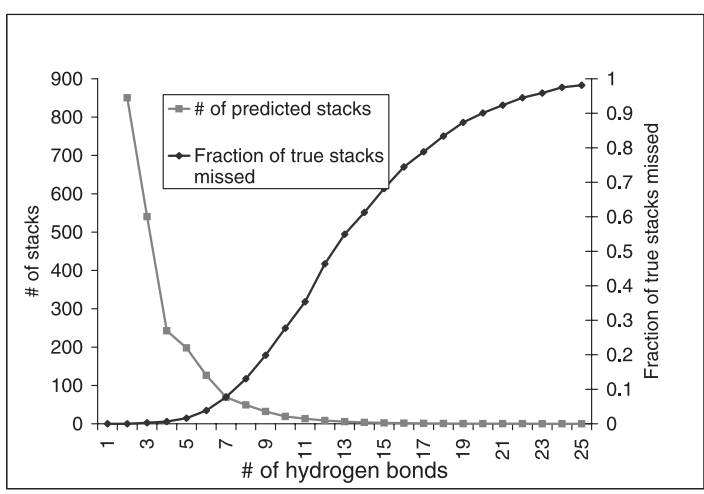

(a)

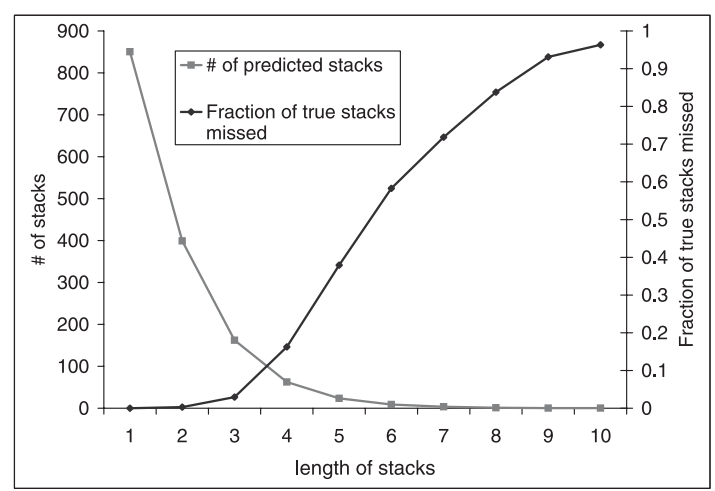

(b)

FIG. 2. Statistics of the stacks in Rfam database. (a) One line shows the fraction of annotated stacks which would be missed outby using different cutoffs - the minimum numbers of hydrogen bonds in the stacks. The other line shows the number of predicted stacks per 100 bases from all Rfam seed sequences using the same cutoffs. (b) One line shows the fraction of annotated stacks which would be missed out by using the minimum lengths of the stacks as cutoffs. The other line shows the number of predicted stacks per 100 bases from all Rfam seed sequences using the same cutoffs. 
distributions of stacks according to the minimum number of hydrogen bonds (Fig. 2a), or stack length (Fig. 2b). Additionally, we plotted the number of putative stacks that can be found on RFAM sequences (normalized to a $100 \mathrm{bp}$ region). If all possible base pairs were considered, we see about 900 putative stacks in a $100 \mathrm{bp}$ region. This number grows quadratically as the length of the sequence increases. Instead, by limiting attention to stacks with length greater than 4, and at least eight hydrogen bonds, we have only 34 putative stacks in a $100 \mathrm{bp}$ region. At the same time, we miss only a small fraction of the true stacks. This computation shows that in considering anchors, it is reasonable to restrict attention to longer stacks.

\subsection{Stack configurations}

A putative stack of length 4 can still be found by chance. Ji et al. (2004) and Touzet and Perriquet (2004) both use sequence similarity to further select stacks. We propose to select a set of stacks instead of one at a time. We evaluate the set of stacks by both the stability (free energy) of the structure they form and the sequence similarity computed based on these common stacks as anchors. To describe the evaluation function of the selected set, we must define a notion of configuration of stacks. Note that two stacks $P_{1}$ and $P_{2}$ may have one of the following relations (Bouthinon and Soldano, 1999): (1) $P_{1}$ and $P_{2}$ are interleaving; (2) $P_{1}$ is enclosed within $P_{2}$ (denoted by $P_{1}<_{I} P_{2}$ ); (3) $P_{1}$ is juxtaposed to $P_{2}$ so that its right end-point is before the left end-point of $P_{2}\left(P_{1}<_{p} P_{2}\right)$. An RNA structure $R_{A}$ on $A$ is a collection of noninterleaving stacks on $A$. The stacks have a partial order $\left(<_{I} \cup<_{p}\right)$ defined on them. The free energy of the structure is the sum of free energies of each of the constituent hairpins, stem-loops, interior loops, and multiloops. Here, we work with the notion of generalized stacks instead of stem-loops. The energy of a stack $P$ denoted by $\mathcal{E}_{s}(P)$ is as described in Equation (1). The energies of loops $\mathcal{E}_{h}$ (hairpin), $\mathcal{E}_{b}$ (bulge), and $\mathcal{E}_{m}$ (multiloops) are a function of the length of the sequence and set as described in Jaeger et al. (1989).

Define a consensus structure $\mathcal{P}(A, B)$ as a pair of structures $R_{A}$ and $R_{B}$ on $A$ and $B$, respectively, with a one-one correspondence between stacks in $R_{A}$ and $R_{B}$ such that the corresponding stacks in the two structures maintain identical partial order relationships. We define the free energy $\phi(\mathcal{P}(A, B))$ of the consensus structure similar to the energy of the individual structures. For each pair of corresponding stacks, or loops, the maximum of the two energy values is chosen.

Given a consensus $\mathcal{P}(A, B)$, the sequences $A$ and $B$ can be aligned to be consistent with $\mathcal{P}(A, B)$ (see Fig. 1c), so that the sequences in stacks are aligned to each other, and likewise for the sequences in the unpaired regions. This alignment partitions sequence $A$ and $B$ into alternating stack and nonstack regions $A_{1}, A_{2}, \ldots, A_{k}$ and $B_{1}, B_{2}, \ldots, B_{k}$. Each pair of sequence $\left(A_{i}, B_{i}\right)$ is aligned optimally. We define such an alignment as a configuration. The cost of the configuration $(A, B, \mathcal{P}(A, B))$ is defined as a function of sequence similarity and the energy of the consensus structure. Denote $i \in \mathcal{P}(A, B)$ if and only if $\left(A_{i}, B_{i}\right)$ are paired in a stack with some $\left(A_{j}, B_{j}\right)$ in $\mathcal{P}(A, B)$. Let $\mathcal{S}\left(A_{i}, B_{i}\right)$ denote the cost of an optimal global alignment of subsequences $A_{i}$ and $B_{i}$. The cost of the configuration $(A, B, \mathcal{P}(A, B))$ is denoted by

$$
M(\mathcal{P}(A, B))=w_{1} \Phi(\mathcal{P}(A, B))+w_{2} \sum_{i \in \mathcal{P}(A, B)} \mathcal{S}\left(A_{i}, B_{i}\right)+w_{3} \sum_{i \notin \mathcal{P}(A, B)} \mathcal{S}\left(A_{i}, B_{i}\right)
$$

where $w_{1}+w_{2}+w_{3}=1$ represent parameters describing the relative weights to the free energy of the configuration, sequence similarity in stack regions, and sequence similarity within loop regions. Ideally, these weights should be adjusted according to the number and divergence of the given sequences. However, in the tests through this paper, we use an identical set of weights $\left(w_{1}=0.84, w_{2}=0.06\right.$, and $\left.w_{3}=0.1\right)$. For a given pair, we compute consensus structures of minimum cost.

The definition of a configuration of stacks for a pair of sequences also extends to multiple sequences: A configuration $\mathcal{P}\left(A_{1}, A_{2}, \ldots, A_{s}\right)$ is a collection of $s$ RNA structures $\left\{P^{A_{1}}, P^{A_{2}}, \ldots, P^{A_{s}}\right\}$, one for each sequence with the following property: For each pair of structures, there is a one-one correspondence between the stacks that is consistent with the partial orders $<_{I}$ and $<_{p}$. A configuration with $l$ stacks partitions each sequence $A_{i}$ into $2 l+1$ blocks denoted $A_{i, 1}, A_{i, 2}, \ldots, A_{i, 2 l+1}$, where each block $A_{*, j}$ is either a stack in the configuration $(j \in \mathcal{P})$ or part of the loop region. We modify Equation (2) to describe 
the cost of the configuration $\mathcal{P}\left(A_{1}, A_{2}, \ldots, A_{s}\right)$ as follows.

$$
\begin{gathered}
M\left(\mathcal{P}\left(A_{1}, \ldots, A_{s}\right)\right)=w_{1} \Phi\left(\mathcal{P}\left(A_{1}, \ldots, A_{s}\right)\right)+w_{2} \sum_{j \in \mathcal{P}\left(A_{1}, \ldots, A_{s}\right)} \mathcal{S}\left(\begin{array}{l}
A_{1, j}, \\
A_{2, j} \\
\ldots, \\
A_{s, j}
\end{array}\right) \\
+w_{3} \sum_{j \notin \mathcal{P}\left(A_{1}, \ldots, A_{s}\right)} \mathcal{S}\left(\begin{array}{l}
A_{1, j}, \\
A_{2, j}, \\
\ldots, \\
A_{s, j}
\end{array}\right)
\end{gathered}
$$

Here, the function $\mathcal{S}$ computes the score of a multiple alignment. The RNA stack based consensus folding problem can be described formally: given $s$ RNA sequences, compute a minimum cost stack configuration. In the following section, we describe algorithms for computing optimal configurations.

\section{STACK-BASED CONSENSUS FOLDING}

\subsection{Computing optimal stack configuration in two RNA sequences}

We use dynamic programming to compute an optimal configuration. The algorithm is similar to prior work (Sankoff, 1985; Bafna et al., 1995) with an important difference being that stacks (instead of individual base pairs) are now used. Given sequences $A, B$, we compute all potential stacks in them, using the algorithm from Section 2.1. Assume these two sequences have $m$ and $n$ stacks, respectively. Let $\mathcal{P}^{A}=$ $P_{1}^{A}, P_{2}^{A}, \ldots, P_{m}^{A}$ and $\mathcal{P}^{B}=P_{1}^{B}, P_{2}^{B}, \ldots, P_{n}^{B}$ denote the stacks, ordered according to increasing values of the right-most base pair. Denote the index of the first and last base pair of a stack $\mathrm{P}$ as $P_{b}, P_{e}$, and the length as $P_{l}$. Define the following terms:

- $\operatorname{Seq}\left(P^{A}\right)$ : The subsequence covered by the stack $P^{A}$, given by $A\left[P_{b}^{A} \ldots P_{b}^{A}+P_{l}^{A}-1\right]$ and $A\left[P_{e}^{A}-\right.$ $\left.P_{l}^{A}+1 \ldots P_{e}^{A}\right]$.

- $\operatorname{Loop}\left(P^{A}\right)$ : The subsequence covered by the first and last positions of the stack $P^{A}$ after excluding the bases in $\operatorname{Seq}\left(P^{A}\right)$. In other words, the sequence $A\left[P_{b}^{A}+P_{l}^{A} \ldots P_{e}^{A}-P_{l}^{A}\right]$.

- $\operatorname{Loop}\left(P^{A}, P^{A^{\prime}}\right)$ : If $P^{A^{\prime}}$ is enclosed within $P^{A}$, then the loop region corresponds to the sequence in between the two stacks (i.e., the subsequences $A\left[P_{b}^{A}+P_{l}^{A} \ldots P_{b}^{A^{\prime}}-1\right]$ and $A\left[P_{e}^{A^{\prime}}+1 \ldots P_{e}^{A}-P_{l}^{A}\right]$ ). If $P^{A^{\prime}}$ is to the left of $P^{A}$, the loop region corresponds to $A\left[P_{e}^{A^{\prime}}+1 \ldots P_{b}^{A}-1\right]$. Otherwise, the term is undefined.

- $M\left[P^{A}, P^{B}\right]$ : The cost of an optimum configuration of $A$ and $B$ over all consensus structures, given that stacks $P^{A}$ and $P^{B}$ are in the consensus structure and aligned to each other.

Clearly, it is sufficient to compute $M\left[P^{A}, P^{B}\right]$ for all pairs in $\mathcal{P}^{A} \times \mathcal{P}^{B}$, which would need $O\left(m^{2} n^{2}\right)$ time. In computing $M\left[P^{A}, P^{B}\right]$, we have three choices for the subsequences $\operatorname{Loop}\left(P^{A}\right)$ and $\operatorname{Loop}\left(P^{B}\right)$, as they could either form a hairpin, an interior loop/bulge, or a multiloop. Therefore,

$$
M\left[P^{A}, P^{B}\right]=M_{s}\left[P^{A}, P^{B}\right]+\min \left\{\begin{array}{ll}
M_{h}\left[P^{A}, P^{B}\right], & (* \text { hairpin loop *) } \\
M_{b}\left[P^{A}, P^{B}\right], & (* \text { interior loop/bulge *) } \\
M_{m}\left[P^{A}, P^{B}\right] & (* \text { multi-loop *) }
\end{array}\right\}
$$

Here, $M_{S}\left[P^{A}, P^{B}\right]$ is the score matching stacks $P^{A}$ and $P^{B}$, based on sequence and structure conservation, and can be computed by

$$
M_{S}\left[P^{A}, P^{B}\right]=w_{1} \max \left\{\begin{array}{l}
\mathcal{E}_{S}\left(P^{A}\right), \\
\mathcal{E}_{S}\left(P^{B}\right)
\end{array}\right\}+w_{2} \mathcal{S}\left(\begin{array}{l}
\operatorname{Seq}\left(P^{A}\right) \\
\operatorname{Seq}\left(P^{B}\right)
\end{array}\right)
$$


$M_{h}\left[P^{A}, P^{B}\right]$ is the score of the loop regions of $P^{A}$ and $P^{B}$ given that no other matched stack pair is included by $P^{A}$ and $P^{B}$, i.e., these regions form matched hairpin loops.

$$
M_{h}\left[P^{A}, P^{B}\right]=w_{1} \max \left\{\begin{array}{c}
\mathcal{E}_{h}\left(\left|\operatorname{Loop}\left(P^{A}\right)\right|\right), \\
\mathcal{E}_{h}\left(\left|\operatorname{Loop}\left(P^{B}\right)\right|\right)
\end{array}\right\}+w_{3} \mathcal{S}\left(\begin{array}{c}
\operatorname{Loop}\left(P^{A}\right), \\
\operatorname{Loop}\left(P^{B}\right)
\end{array}\right)
$$

$M_{b}\left[P^{A}, P^{B}\right]$ represents the matching score when $P^{A}$ and $P^{B}$ are followed by an interior loop or bulge. Consider all stacks $P^{x}, P^{y}$ that are enclosed by $P^{A}$ and $P^{B}$, respectively. Then, $M_{b}\left[P^{A}, P^{B}\right]$ is the minimum free energy of any matching of $P^{x}, P^{y}$.

$$
\begin{aligned}
M_{b}\left[P^{A}, P^{B}\right]=\min _{P^{x}<_{I} P^{A}} & \left\{w_{1} \max \left\{\begin{array}{l}
\mathcal{E}_{b}\left(\left|\operatorname{Loop}\left(P^{x}, P^{A}\right)\right|\right), \\
\mathcal{E}_{b}\left(\left|\operatorname{Loop}\left(P^{y}, P^{B}\right)\right|\right)
\end{array}\right\}\right. \\
& \left.+w_{3} \mathcal{S}\left(\begin{array}{l}
\operatorname{Loop}\left(P^{x}, P^{A}\right), \\
\operatorname{Loop}\left(P^{y}, P^{B}\right)
\end{array}\right)+M\left[P^{x}, P^{y}\right]\right\}
\end{aligned}
$$

For the multiloop case, we need to define some additional terms. A sequence of stacks $P_{1}, P_{2}, \ldots$ form a chain if $P_{1}<_{p} P_{2}<_{p} \ldots M_{m}\left[P^{A}, P^{B}\right]$ represents the matching score between $P^{A}$ and $P^{B}$, given that there is a pair of chains included by $P^{A}$ and $P^{B}$ which form the multiloop. Let $P_{1}^{A}, P_{2}^{A}, \ldots$ (respectively, $\left.P_{1}^{B}, P_{2}^{B}, \ldots\right)$ denote stacks enclosed by $P^{A}\left(P^{B}\right.$, respectively) and ordered according to increasing values of the last coordinate. Denote $P_{i_{1}}^{A} \in F\left(P_{i_{2}}^{A}\right)$ if $P_{i_{1}}^{A}<_{p} P_{i_{2}}^{A}$ and there is no stack $P_{j}^{A}$ such that $P_{i_{1}}^{A}<_{p} P_{j}^{A}<_{p} P_{i_{2}}^{A}$. Then,

$$
M_{m}\left[P^{A}, P^{B}\right]=\min _{i, j}\left\{M_{c}\left[P_{i}^{A}, P_{j}^{A}\right]+w_{1} \max \left\{\begin{array}{l}
\mathcal{E}_{m}\left(\left|\operatorname{Loop}\left(P^{i}, P^{A}\right)\right|\right), \\
\mathcal{E}_{m}\left(\left|\operatorname{Loop}\left(P^{j}, P^{B}\right)\right|\right)
\end{array}\right\}+w_{3} \mathcal{S}\left(\begin{array}{l}
\operatorname{Loop}\left(P^{i}, P^{A}\right), \\
\operatorname{Loop}\left(P^{j}, P^{B}\right)
\end{array}\right)\right\} .
$$

Here, $M_{c}\left[P_{i}^{A}, P_{j}^{B}\right]$ is defined as the minimum energy of a chain that ends at $P_{i}^{A}$, and $P_{j}^{B}$ and begins at some $P_{i^{\prime}}^{A}<{ }_{p} P_{i}^{A}$, and $P_{j^{\prime}}^{B}<{ }_{p} P_{j}^{B}$. (The end conditions are added for efficiency reasons.) Then,

$$
M_{c}\left[P_{i}^{A}, P_{j}^{B}\right]=\min _{\substack{P_{x}^{A} \in F\left(P_{i}^{A}\right) \\
P_{y}^{A} \in F\left(P_{i}^{B}\right)}}\left\{\begin{array}{l}
M_{c}\left[P_{x}^{A}, P_{y}^{B}\right]+M_{o}\left[P_{x}^{A}, P_{i}^{A} ; P_{y}^{B}, P_{j}^{B}\right]+M\left[P_{i}^{A}, P_{j}^{B}\right], \\
M_{c}\left[P_{i}^{A}, P_{y}^{B}\right]+\mathcal{E}_{m}\left(\left|\operatorname{Loop}\left(P_{x}^{A}, P_{i}^{A}\right)\right|+\left|\operatorname{Seq}\left(P_{i}^{A}\right)\right|\right), \\
M_{c}\left[P_{x}^{A}, P_{j}^{B}\right]+\mathcal{E}_{m}\left(\left|\operatorname{Loop}\left(P_{y}^{B}, P_{j}^{B}\right)\right|+\left|\operatorname{Seq}\left(P_{j}^{B}\right)\right|\right)
\end{array}\right\}
$$

where $M_{o}\left[P_{x}^{A}, P_{i}^{A} ; P_{y}^{B}, P_{j}^{B}\right]$ is the minimum free energy of the matching between the loops $\left(P_{x}^{A}, P_{i}^{A}\right)$ and $\left(P_{y}^{B}, P_{j}^{B}\right)$,

$$
M_{o}\left[P_{x}^{A}, P_{i}^{A} ; P_{y}^{B}, P_{j}^{B}\right]=w_{1} \max \left\{\begin{array}{l}
\mathcal{E}_{b}\left(\left|\operatorname{Loop}\left(P_{x}^{A}, P_{i}^{A}\right)\right|\right), \\
\mathcal{E}_{b}\left(\left|\operatorname{Loop}\left(P_{y}^{B}, P_{j}^{B}\right)\right|\right)
\end{array}\right\}+w_{3} \mathcal{S}\left(\begin{array}{l}
\operatorname{Loop}\left(P_{x}^{A}, P_{i}^{A}\right), \\
\operatorname{Loop}\left(P_{y}^{B}, P_{j}^{B}\right)
\end{array}\right) .
$$

\subsection{Consensus fold computation for multiple RNA sequences}

The optimal configuration of a randomly chosen pair of sequences from a family already shows high sensitivity (data not shown). It is likely that an optimal configuration of structures conserved in diverse multiple sequences will be very accurate. Recall the cost of the configuration as Equation (3), where $\mathcal{S}$ denotes the score of a multiple alignment of the block. Clearly, the problem of computing optimal configuration is hard, given the discussion for the pairwise case. Here, we use a heuristic principle based on the notion of a star-alignment, with a seed configuration chosen from an optimal configuration of a random pair of sequences. To understand why our approach should work, we describe a back-of-theenvelope calculation.

Consider a stack $x$ of length $k$ from the seed structure defined on sequence $A_{1}$ that is in fact incorrect (does not overlap with a true stack). For $x$ to be retained in the final anchored configuration, it must match with stacks in a large fraction of the other sequences. Let $p$ be the probability that a random pair of bases 
Table 1. EfFect of Parameters $k, w$, and $s$ ON The Probability of Predicting Conserved Stacks at Random ${ }^{\mathrm{a}}$

\begin{tabular}{llll}
\hline$k$ & $w$ & $s$ & \multicolumn{1}{c}{$P_{c}(x)$} \\
\hline 4 & 10 & 20 & 0.0008 \\
4 & 40 & 20 & 0.13 \\
4 & 80 & 20 & 0.91 \\
5 & 80 & 20 & 0.02 \\
6 & 80 & 20 & $1.8 \mathrm{e}-6$ \\
5 & 80 & 40 & 0.001 \\
5 & 80 & 60 & $7.35 \mathrm{e}-05$
\end{tabular}

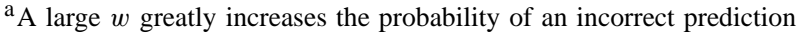
Waterman (1989) has performed similar statistical analysis.

can form base pairs. Given an interval $(i, j)$ in some sequence, the probability that $(i, j)$ is the end of a stack of length $k$ is $p^{k}$. The probability that $x$ is matched up with some random set of base pairs defined by the end-points $(i, j)$ is no more than $p^{k}$, even after ignoring sequence similarity. However, $x$ cannot be matched to any other arbitrary stack. As we also score for primary sequence conservation, and the match should maintain the partial order of the configuration, $(i, j)$ and $x$ must be "similarly situated." To model this, we introduce a parameter $w$. Define $w$ as the number of distinct pairs $(i, j)$ such that $x$ is allowed to match. Then, the probability that $x$ finds a match by chance is given by $p_{x}=1-\left(1-p^{k}\right)^{w}$. Allowing a more flexible definition, we say that $x$ is $f$-conserved in the configuration if it finds a match in at least $(1-f) s$ of the $s$ sequences.

$$
\operatorname{Pr}[x \text { is } f \text {-conserved }]=P_{c}(x)=\sum_{l \geq(1-f) s}\left(\begin{array}{l}
s \\
l
\end{array}\right) p_{x}^{l}\left(1-p_{x}\right)^{s-l}
$$

Fix some parameters as follows. Let $p=\frac{3}{8}$ (corresponding to G-C, G-U, A-U), $f=0.7$. Table 1 describes the impact on $P_{c}(x)$ for varying values of $k, s, w$. The probability of getting an incorrect conserved stack depends critically on the parameters $w$. If $w$ is too large, there is a high probability of getting random stacks to match up. This effect might be offset by increasing $k$, but then we risk losing many true (smaller size) stacks, which may cause incorrect pairs to be matched. The effect is also offset (to a less degree) by increasing the number of sequences, but that may not always be possible. The reason our approach works is because the choice of a conserved configuration restricts the possible stacks that $x$ can match to, effectively keeping the value of $w$ low.

Before describing the approach, we must first modify the formulation to allow stacks to be partially conserved and, therefore, absent from some sequences. For $0<f \leq 1$, define an $f$-configuration as a configuration with the following property: for every set of $s$ corresponding stacks (one from each sequence),

Procedure COMPUTEANChorCONFIGURATION $(k, f)$

1. Pick a pair of sequences $(A, B)$ at random from the set $\mathcal{R}$ of RNA sequences.

2. Compute putative stacks $\mathcal{P}^{A}$ from $A$, and $\mathcal{P}^{B}$ from $B$ with minimum length $k$. $k$ is chosen according to the lengths of sequences in $\mathcal{R}$, and typically $k=4$.

3. Compute the optimum configuration. Reduce $\mathcal{P}^{A}$ to retain only the stacks from the optimum configuration. Denote $\mathcal{P}^{A}$ as the reduced set.

4. For each sequence $R \in \mathcal{R}$, compute the optimum pairwise configuration of $(A, R)$ using the reduced set $\mathcal{P}^{\prime A}$. Denote $M[(A, B), \mathcal{R}]$ as the sum of the configuration costs.

5. Recompute Steps $1-4$ for various random choices of $(A, B)$, and pick the pair $(A, B)$ with minimum configuration cost $M[(A, B), \mathcal{R}]$.

6. Retain only the stacks in $\mathcal{P}^{\prime A}$ that appear in $1-f$ fraction of the sequences in $\mathcal{R}$. Denote the subset as $\mathcal{P}^{\prime \prime}$. Output $\mathcal{P}^{\prime \prime}$ as the anchored structure of $\mathcal{R}$.

FIG. 3. The procedure for computing anchor configuration. 


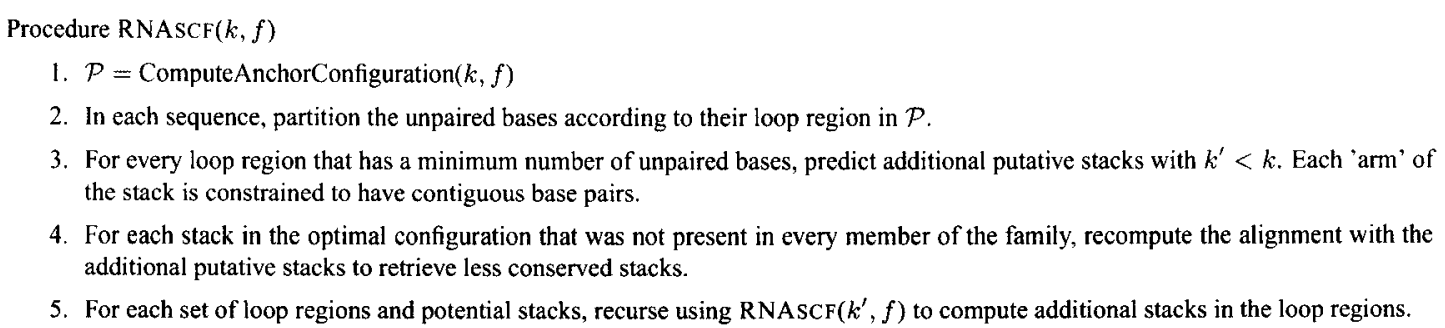

FIG. 4. The procedure RNAscf for computing consensus folds.

at most $(1-f) s$ can be absent. In Fig. 3, we describe a procedure for computing an anchor configuration. The anchor configuration consists of stacks that optimize the cost of the configuration and are conserved across the family. Thus, the stacks are highly likely to be correct. However, the procedure might also miss some true stacks due to a high initial value of $k$ and requirement of conservation. To increase sensitivity, we now search for less conserved and shorter stacks. However, the new stacks are forced to be consistent with the anchor configuration.

Recall from the definition of loops in Section 2.1 that all unpaired bases can be uniquely assigned to a loop. Additionally, a stack does not interleave with the anchor configuration if and only if it is defined on unpaired bases within a single loop. This forms the basis of the final procedure RNAscf. See Fig. 4.

\subsection{Implementation details}

The program (RNAscf) is implemented in $\mathrm{C}$ and is available upon request. In the default setting, we limit ourselves to two iterations. For the first iteration, we choose the default parameters as $k=4, h=8$ ( $k$ is the minimum length and $h$ is the minimum number of hydrogen bonds in the putative stacks), and $g=0$ (no unpaired bases allowed in stacks). For the second iteration, the default settings are changed to $k=3, h=6$.

\section{RESULTS AND DISCUSSION}

To test the performance of RNAscf, we chose a set of 12 RNA families from the Rfam database (Griffiths-Jones et al., 2003). Twenty sequences were chosen for each family, except for CRE (RF00220) and $\operatorname{glmS}$ (RF00234) for which we chose available 10 sequences, respectively. Stacks were retrieved from the annotated structures for each of these sequences. In all, there are 953 stacks. We chose three other programs to compare the performance of RNAscf, choosing the best representative of different methodologies: RNAfold, which is an implementation of energy-based minimization (Jaeger et al., 1989) from the Vienna package (Hofacker, 2003); COVE, which is an implementation of covariance model (Eddy and Durbin, 1994), and comRNA (Ji et al., 2004), which is based on computing anchors in multiple sequence alignment. Only comRNA predicts stacks explicitly. COVE and RNAfold do not explicitly predict stacks, but most of their base pairs appear in stacks. For best results, we ignored unstacked base pairs (with unpaired bases on either side) for RNAfold and COVE. Larger stack length cutoffs were also tried, but this choice gave the best balance of sensitivity and accuracy. Sensitivity is defined as the fraction of true stacks that overlapped with predicted stacks. A sensitivity of 1 would imply that all true stacks overlapped with some predicted stacks. Correspondingly accuracy is the fraction of predicted stacks that overlapped with a true stack. As COVE expects aligned sequences, we aligned the sequences using ClustalW (Thompson et al., 1994). The alignment was used to train the covariance model, and the model was then used to align sequences, and predict structure. We also ran COVE on unaligned sequences, but the performance in that case was inferior to the performance on ClustalW aligned sequences.

Figure 5 shows the plots of the sensitivity and specificity of all programs on the test (detailed numbers are shown in Table 2). As can be seen in the tables, RNAscf is at the top or near the top in every family and maintains high sensitivity and accuracy throughout, with an average accuracy of 0.884 and average sensitivity of 0.926 . Only comRNA shows consistently high accuracy because it predicts very few stacks 


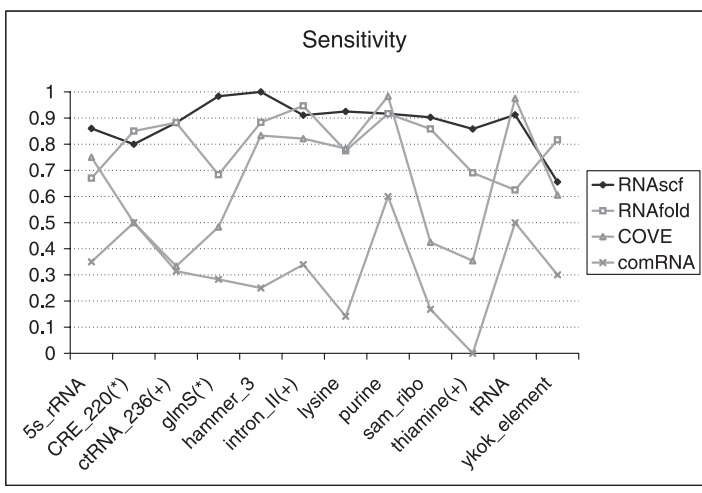

(a)

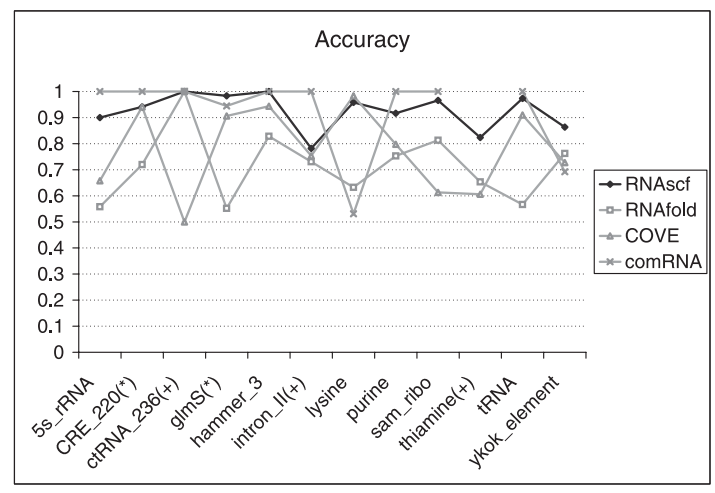

(b)

FIG. 5. Sensitivity and accuracy of RNA secondary structure prediction on 12 RNA families. The default parameters for RNAscf are $f=0.7, k=4$, and $h=8$ for the first iteration. RNAfold is run under default parameters. COVE is run under the default parameters, using the multiple alignment from ClustalW as input while comRNA is run under the recommended parameter $(p=0.7$ and $s=0.56)$. $(*)$ There are only 10 sequences in these families. $(+)$ RNAscf is run under $k=3$ and $h=6$ on these families, due to their small size.

(leading to low sensitivity) that are well conserved in both sequence and structure. COVE occasionally shows very poor sensitivity, possibly because of incorrect seed alignment. RNAfold predicts many stacks and therefore has good sensitivity, but the extra predictions lead to loss of accuracy. While our method shows robust performance for a limited number of given RNA sequences, its performance improves when the number of the given sequences increase. Figure 6 shows the sensitivity and accuracy as the number of sequences increase for the thiamine subfamily. Both the sensitivity and accuracy exceed 0.9 when $s=80$. Similar results were obtained for other large families.

Finally, we emphasize that even though sometimes we cannot get all the stacks in all the given sequences, the consensus structure obtained by RNAscf is always the right configuration; the prediction errors in a few input sequences are usually due to an incorrect stack that is very close to a correct one. Since this cannot be quantified, we use one example to demonstrate that the minor prediction error in a few given sequences does not affect the prediction of the common structure. Figure 7 shows the predicted configuration of the four programs on the SAM riboswitch. Clearly, RNAfold can predict the correct configuration in some of

Table 2. A Complete List of the Comparison of Sensitivity and Accuracy of RNA Secondary Structure Prediction on 12 RNA Families Shown in Fig. 5

\begin{tabular}{|c|c|c|c|c|c|c|c|c|c|}
\hline \multirow[b]{2}{*}{ Name (Rfam_id) } & \multirow[b]{2}{*}{ Stacks } & \multicolumn{4}{|c|}{ Sensitivity } & \multicolumn{4}{|c|}{ Accuracy } \\
\hline & & RNAscf & RNAfold & Cove & $\operatorname{comRNA}$ & RNAscf & RNAfold & Cove & comRNA \\
\hline 5s_rRNA (RF00001) & 100 & 0.86 & 0.67 & 0.75 & 0.35 & 0.9 & 0.558 & 0.658 & 1 \\
\hline Rhino_CRE (RF00220) & 20 & 0.8 & 0.85 & 0.5 & 0.5 & 0.941 & 0.72 & 0.941 & 1 \\
\hline ctRNA_pGA1 (RF00236) & 51 & 0.882 & 0.882 & 0.333 & 0.313 & 1 & 1 & 0.5 & 1 \\
\hline $\operatorname{glmS}(\mathrm{RF} 00234)$ & 60 & 0.983 & 0.683 & 0.483 & 0.283 & 0.983 & 0.552 & 0.906 & 0.944 \\
\hline Hammerhead_3 (RF00008) & 60 & 1 & 0.883 & 0.833 & 0.25 & 1 & 0.828 & 0.943 & 1 \\
\hline Intron_gpII (RF00029) & 56 & 0.91 & 0.946 & 0.821 & 0.339 & 0.782 & 0.731 & 0.754 & 1 \\
\hline Lysine (RF00168) & 120 & 0.925 & 0.775 & 0.783 & 0.142 & 0.958 & 0.633 & 0.984 & 0.531 \\
\hline Purine (RF00167) & 60 & 0.917 & 0.917 & 0.983 & 0.6 & 0.917 & 0.753 & 0.797 & 1 \\
\hline Sam_riboswitch (RF00162) & 113 & 0.903 & 0.858 & 0.425 & 0.168 & 0.966 & 0.813 & 0.613 & 1 \\
\hline Thiamine (RF00059) & 80 & 0.858 & 0.690 & 0.354 & 0 & 0.824 & 0.654 & 0.606 & - \\
\hline tRNA (RF00005) & 113 & 0.912 & 0.625 & 0.975 & 0.5 & 0.973 & 0.567 & 0.910 & 1 \\
\hline ykok (RF00380) & 180 & 0.656 & 0.817 & 0.606 & 0.3 & 0.863 & 0.762 & 0.727 & 0.692 \\
\hline Average & & 0.884 & 0.8 & 0.654 & 0.312 & 0.926 & 0.714 & 0.778 & 0.924 \\
\hline
\end{tabular}




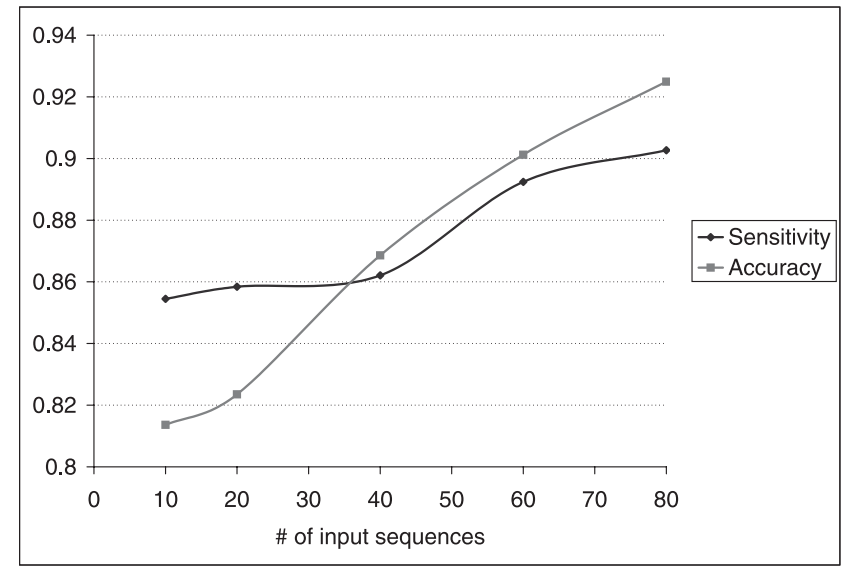

FIG. 6. Improved sensitivity and accuracy of RNAscf as the number of input sequences grows for the thiamine family.

the RNA sequences, but can make the wrong prediction on the others. This is not surprising, because it analyzes each RNA sequence separately and doesn't presume they have the common structure. However, it will be difficult to derive their common structure based on these results. comRNA tends to miss many real stacks, although the ones it predicts are often correct. COVE predicted some correct stacks but it may miss some correct stacks and may also predict some wrong ones. Similar results were seen for all families.

In conclusion, RNAscf establishes the principle that anchored stacks selection based on seed configurations and prediction of consensus structure subject to anchored constraints are a valid approach to RNA structure prediction. Our future work will be aimed at correcting errors by using a stochastic iterative scheme such as Gibbs sampling (Lawrence et al., 1993). In each step, we will remove a stack from the consensus structure and add a new stack sampled from possibilities that are consistent with the remaining configuration and weighted according to the energy. Early experiments have shown the promise of such refinement.

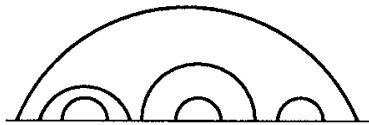

(a)

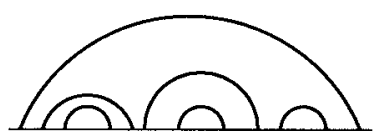

(d1)

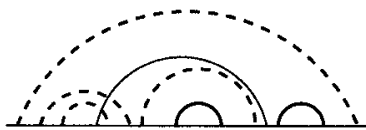

(el)

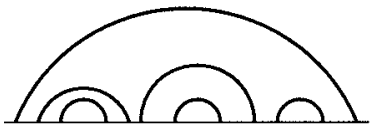

(b)

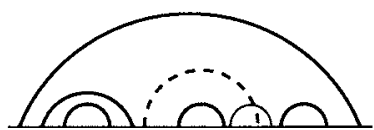

(d2)

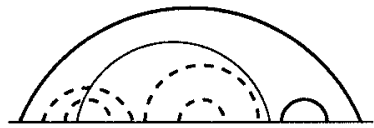

(e2)

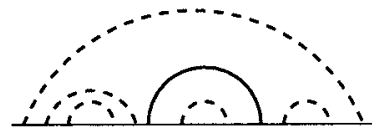

(c)

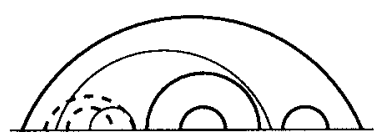

(d3)

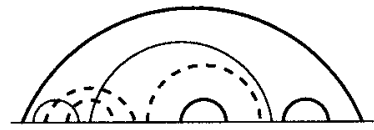

(e3)

FIG. 7. A comparison of predicted stack configurations by different programs. (a) The true consensus stack configuration for the sam riboswitch (RF00162). (b) RNAscf prediction. (c) comRNA prediction. (d1)-(d3) The first three RNAfold predictions. (e1)-(e3) The first three COVE predictions. Note that RNAfold and COVE are not limited to predicting conserved stack configuration, and, therefore, give potentially a different answer for each sequence. Thick line, dashed line and thin line represent true stacks, missed stacks, and wrong stacks in the corresponding predicted configurations. 


\section{ACKNOWLEDGMENT}

This research was funded by a grant from the National Science Foundation NSF-DBI:0516440 (S.Z. and V.B.).

\section{REFERENCES}

Argaman, L., Hershberg, R., Vogel, J., Bejerano, G., Wagner, E.G., Margalit, H., et al. 2001. Novel small RNA-encoding genes in the intergenic regions of Escherischia coli. Curr. Biol. 11, 941-950.

Bafna, V., Muthukrishnan, S., and Ravi, R. 1995. Computing similarity between RNA strings. Combinatorial Pattern Matching 937, 1-14.

Bouthinon, D., and Soldano, H. 1999. A new method to predict the consensus secondary structure of a set of unaligned RNA sequences. Bioinformatics 15, 785-798.

Bray, N., and Pachter, L. 2004. MAVID: Constrained ancestral alignment of multiple sequences. Genome Res. 14, 693-699.

Brudno, M., Poliakov, A., Salamov, A., Cooper, G., Sidow, A., Rubin, E., et al. 2004. Automated whole-genome multiple alignment of rat, mouse, and human. Genome Res. 14, 685-692.

Cawley, S., Bekiranov, S., Ng, H.H., Kapranov, P., Sekinger, E.A., Kampa, D., et al. 2004. Unbiased mapping of transcription factor binding sites along human chromosomes 21 and 22 points to widespread regulation of noncoding RNAs. Cell 116, 499-509.

Davydov, E., and Batzoglou, S. 2004. A computational model for RNA multiple structural alignment. Combinatorial Pattern Matching 3109, 254-269.

Eddy, S.R. 2001. Non-coding RNA genes and the modern RNA world. Nature Rev. Genet. 2, 919-929.

Eddy, S.R., and Durbin, R. 1994. RNA sequence analysis using covariance models. Nucl. Acids Res. 22, $2079-2088$.

Gorodkin, J., Heyer, L.J., and Stormo, G.D. 1997. Finding the most significant common sequence and structure motifs in a set of RNA sequences. Nucl. Acids Res. 25, 3724-3732.

Gorodkin, J., Stricklin, S.L., and Stormo, G.D. 2001. Discovering common stem-loop motifs in unaligned RNA sequences. Nucl. Acids Res. 29, 2135-2144.

Griffiths-Jones, S., Bateman, A., Marshall, M., Khanna, A., and Eddy, S.R. 2003. Rfam: An RNA family database. Nucl. Acids Res. 31, 439-441.

Hofacker, I.L. 2003. Vienna RNA secondary structure server. Nucl. Acids Res. 31, 3429-3431.

Hofacker, I.L., Fekete, M., and Stadler, P.F. 2002. Secondary structure prediction for aligned RNA sequences. J. Mol. Biol. 319, 1059-1066.

Hofacker, I.L., Fontana, W., Stadler, P.F., Bonhoeffer, L.S., Tacker, M., and Schuster, P. 1994. Fast folding and comparison of RNA secondary structures. Monatsh. Chem. 125, 167-188.

International, H.G.S.C. 2004. Finishing the euchromatic sequence of the human genome. Nature 431, 931-945.

Jaeger, J.A., Turner, D.H., and Zuker, M. 1989. Improved predictions of secondary structures for RNA. Proc. Natl. Acad. Sci. USA 86, 7706-7710.

Ji, Y., Xu, X., and Stormo, G.D. 2004. A graph theoretical approach for predicting common RNA secondary structure motifs including pseudoknots in unaligned sequences. Bioinformatics 20, 1591-1602.

Kampa, D., Cheng, J., Kapranov, P., Yamanaka, M., Brubaker, S., Cawley, S., et al. 2004. Novel RNAs identified from an in-depth analysis of the transcriptome of human chromosomes 21 and 22. Genome Res. 14, 331-342.

Knight, R., Birmingham, A., and Yarus, M. 2004. BayesFold: Rational 2 degrees folds that combine thermodynamic, covariation, and chemical data for aligned RNA sequences. RNA 10, 1323-1336.

Knudsen, B., and Hein, J. 2003. Pfold: RNA secondary structure prediction using stochastic context-free grammars. Nucl. Acids Res. 31, 3423-3428.

Lawrence, C.E., Altschul, S.F., Boguski, M.S., Liu, J.S., Neuwald, A.F., and Wootton, J.C. 1993. Detecting subtle sequence signals: A Gibbs sampling strategy for multiple alignment. Science 262, 208-214.

Levitt, M. 1969. Detailed molecular model for transfer ribonucleic acid. Nature 224, 759-763.

Lippert, R.A., Zhao, X., Florea, L., Mobarry, C., and Istrail, S. 2004. Finding anchors for genomic sequence comparison. RECOMB, 233-241.

Mathews, D.H., and Turner, D.H. 2002. Dynalign: An algorithm for finding the secondary structure common to two RNA sequences. J. Mol. Biol. 317, 191-203.

Nahvi, A., Sudarshan, N., Ebert, M.S., Zou, X., Brown, K.L., and Breaker, R.R. 2003. Genetic control by a metabolite binding mRNA. Chem. Biol. 9, 1043-1049.

Nussinov, R., and Jacobson, A.B. 1980. Fast algorithm for predicting the secondary structure of single-stranded RNA. Proc. Natl. Acad. Sci. USA 77, 6309-6313. 
Nussinov, R., Pieczenik, G., Griggs, J.R., and Kleitman, D.J. 1978. Algorithms for loop matchings. SIAM J. Appl. Math. 35, 68-82.

Pavesi, G., Mauri, G., Stefani, M., and Pesole, G. 2004. RNAProfile: An algorithm for finding conserved secondary structure motifs in unaligned RNA sequences. Nucl. Acids Res. 32, 3258-3269.

Perriquet, O., Touzet, H., and Dauchet, M. 2003. Finding the common structure shared by two homologous RNAs. Bioinformatics 19, 108-116.

Sakakibara, Y., Brown, M., Hughey, R., Mian, I.S., Sjölander, K., Underwood, R.C., et al. 1994. Recent methods for RNA modeling using stochastic context free grammars. Combinatorial Pattern Matching 807, 289-306.

Sankoff, D. 1985. Simultaneous solution of the RNA folding, alignment and protosequence problems. SIAM J. Appl. Math. 45, 810-825.

Smith, T.F., and Waterman, M.S. 1978. RNA secondary structure. Math. Biosci. 42, 257-266.

Stormo, G.D. 2003. New tricks for an old dogma: Riboswitches as cis-only regulatory systems. Mol. Cell 11, 14191420.

Storz, G. 2002. An expanding universe of noncoding RNAs. Science 296, 1260-1263.

Thompson, J.D., Higgins, D.G., and Gibson, T.J. 1994. CLUSTAL W: Improving the sensitivity of progressive multiple sequence alignment through sequence weighting, position-specific gap penalties and weight matrix choice. Nucl. Acids Res. 22, 4673-4680.

Tinoco, I., Uhlenbeck, O.C., and Levine, M.D. 1971. Estimation of secondary structure in ribonucleic acids. Nature 230, 362-367.

Touzet, H., and Perriquet, O. 2004. CARNAC: Folding families of related RNAs. Nucl. Acids Res. 32, 142-145.

Vitreschak, A.G., Rodionov, D.A., Mironov, A.A., and Gelfand, M.S. 2003. Riboswitches: The oldest mechanism for the regulation of gene expression? Trends Genet. 20, 44-50.

Waterman, M.S. 1978. Secondary structure of single stranded nucleic acids. Adv. Math. Suppl. Stud. I, 167-212.

Waterman, M.S. 1989. Consensus methods for fodling single-stranded nucleic acids. Mathematical Methods for DNA Sequences, 185-224.

Zuker, M. 1994. Prediction of RNA secondary structure by energy minimization. Methods Mol. Biol. 25, $267-294$.

Zuker, M. 2003. Mfold web server for nucleic acid folding and hybridization prediction. Nucl. Acids Res. 31, 34063415.

Zuker, M., and Sankoff, D. 1984. RNA secondary structure and their prediction. Bull. Math. Biol. 46, 591-621.

Zuker, M., and Stiegler, P. 1981. Optimal computer folding of large RNA sequences using thermodynamics and auxiliary information. Nucl. Acids Res. 9, 133-148.

Address correspondence to:

Shaojie Zhang

Dept. of Computer Science and Engineering

University of California, San Diego

La Jolla, CA 92093

E-mail: shzhang@cs.ucsd.edu 